INVESTIGACIONES

\title{
CREENCIAS DEL PROFESORADO UNIVERSITARIO DE EDUCACION FISICA EN RELACION A LOS CONCEPTOS DE CUERPO Y SALUD
}

\author{
Beliefs of university teaching staff's physical education regarding body \\ and health concepts
}

\author{
Alberto Moreno ${ }^{1}$, Nidia Alvarez ${ }^{2}$ \\ ${ }^{1}$ Instituto de Filosofía y Estudios Educacionales. Facultad de Filosofía y Humanidades. \\ Universidad Austral de Chile, Valdivia. Chile, alberto.moreno@docentes.uach.cl \\ ${ }^{2}$ Escuela de Educación. Universidad Santo Tomás, La Serena. Chile, nidiaalvarezdelajara@gmail.com
}

\section{Resumen}

El artículo que a continuación se presenta es el resultado de un trabajo de investigación realizado en una universidad privada de $\mathrm{La}$ Serena, IV Región, Chile, durante el año 2008. Los objetivos de investigación han sido identificar, describir y analizar (interpretativamente) las creencias del profesorado universitario de Educación Física en relación a los conceptos de "Cuerpo" y "Salud", pues dichas creencias condicionan la forma de entender nuestra disciplina y las formas de practicarla y orientar/ guiar nuestro quehacer profesional. La presente investigación es un estudio fenomenológico enmarcado dentro del paradigma interpretativo, cuya metodología es cualitativa dado su perfil descriptivo y relacional. Los resultados obtenidos muestran que, a pesar del desarrollo de la educación física actual, las creencias del profesorado universitario en relación a los dos conceptos mencionados sigue siendo coherente con lo que en la literatura especializada se ha conocido como educación física tecnocrática, con las concepciones de cuerpo y salud acordes a dicha visión.

Palabras clave: creencias, profesorado universitario, educación física, cuerpo, salud.

\begin{abstract}
The following article is the result of an investigation carried out at a private university in La Serena, IV Region, Chile, during the year 2008. The investigation goals have been to identify, describe and analyse (interpretatively) the beliefs of University teaching staff (physical education) regarding the "Body" and "Health" concepts, as those beliefs determine the way our discipline is understood, practiced, and also the way in which our professional daily routines are carried out. This investigation is a phenomenological study formulated inside the interpretative paradigm. The methodology is qualitative due to its descriptive and relational profile. The results of this study show that, despite the development of the physical education discipline, the beliefs of University teaching staff (physical education) regarding the two above mentioned concepts are coherent with what is known in the specialized literature as technocratic physical education. The concepts of body and health are in agreement with the principles of technocratic physical education.
\end{abstract}

Key words: beliefs, University teaching staff, physical education, body, health. 


\section{INTRODUCCION}

Al partir de una concepción de la Educación como espacio de participación, interacción y diálogo se hace indispensable escuchar la voz del profesorado universitario para develar y hacer públicas las creencias que poseen en relación a dos de los conceptos que explícita o implícitamente forman parte de la definición que ellos tienen de Educación Física: estos son el "cuerpo" y la "salud".

Sería bastante complejo no aceptar que la Educación Física trata con el cuerpo y sus distintas manifestaciones motrices, así como que la salud es parte intrínseca de nuestra labor como profesionales del cuerpo y el movimiento. Aceptar afirmaciones como éstas son del todo normales en nuestro ámbito profesional. Lo verdaderamente complejo es ponernos de acuerdo en relación a las concepciones que cada uno de nosotros tenemos de ambos conceptos, pues las miradas, perspectivas y orientaciones académicas y profesionales varían al respecto.

Es por ello que se nos hace relevante realizar este estudio cuyo objeto es conocer las concepciones implícitas del profesorado universitario de educación física en relación a los conceptos de cuerpo y salud, pues dichas creencias van a condicionar la forma de entender nuestra disciplina y las formas de practicarla y orientar/guiar nuestra práctica.

Haciendo una revisión tanto de los conceptos de cuerpo como de salud a lo largo de la historia podemos encontrar que el concepto de cuerpo ha sido entendido, y sigue siéndolo, en función de tres perspectivas determinadas (Pérez y Sánchez, 2001). La primera de ellas tiene relación con entender el cuerpo desde una corriente dualista en donde se separa lo natural de lo cultural, desde donde el cuerpo es básicamente materia y por tanto el estudio del mismo se ha centrado en su realidad biofisiológica. Es desde aquí que surge la ya tan conocida metáfora del cuerpo máquina. La segunda perspectiva tiene relación con las concepciones monistas del cuerpo y que entienden al individuo como una esencialidad integrada en un todo, y que por tanto la vivencia del ser es también corporal y que la corporeidad es algo más que una materialidad ocupada por la mente. Por último, la tercera perspectiva tiene relación con una concepción del cuerpo como construcción sociocultural y que tiene relación con una concepción del cuerpo formada en función de las diferentes características del contexto sociocultural e histórico en donde dicha concepción se desarrolla.

Estas diferentes configuraciones desde donde se explica y se entiende el concepto de cuerpo están muy relacionadas con las perspectivas que para Devís y Peiró (2001: 307-310) existen en las formas de promocionar la salud entre una población determinada. Para estos autores son cinco las principales perspectivas de promoción de la actividad física relacionada con la salud.

a) Perspectiva mecanicista: existe la creencia de que la realización de actividades física produce un hábito de práctica que continuará en el futuro.

b) Perspectiva orientada al conocimiento: se fundamenta en la capacidad de decisión y adquisición de conocimientos por parte de los participantes, ya que pretende ayudarles en la elección y toma de decisiones informadas respecto a la actividad física y la salud. 
c) Perspectiva orienta a las actitudes: fundamenta las decisiones en la percepción e interpretación que hacen las personas de su propia experiencia cuando se implican en actividades físicas.

d) Perspectiva crítica: sitúa los problemas de salud a nivel social y ambiental y, por lo tanto, sus estrategias de promoción se dirigen a facilitar el cambio social y comunitario.

e) Perspectiva ecológica: considera las comunidades humanas como complejos sistemas de interdependencias, análogas a los ecosistemas naturales, y asume que la salud es tanto una responsabilidad personal como un asunto social y ambiental.

Cierto que en los discursos de los profesores se pueden afirmar y/o rechazar algunas de las conceptualizaciones realizadas hasta el momento, entendiendo que es políticamente más correcto sentirse guiado por una concepción del cuerpo como construcción sociocultural y en una perspectiva crítica o ecológica de la promoción de la salud. Pero una cosa es lo que el profesorado pueda decir qué piensa y otra cosa es lo que realmente piensa.

Es por ello que en este trabajo nos ha interesado realizar una investigación acerca de las creencias que los profesores/as universitarios tienen, pues son estas creencias las que de alguna forma orientan y guían sus prácticas, tanto en función de lo que hacen como en función de lo que dejan de hacer.

En este sentido, el estudio de las creencias del profesorado en relación a los conceptos de cuerpo y salud puede tener notables repercusiones, concretamente por dos motivos: primero por la relación que guardan con las prácticas y conductas profesionales, y segundo por su incidencia en la propia visión del estudiante universitario hacia los conceptos que se han mencionado.

Contreras y otros (2002) ponen énfasis en la importancia del estudio de las creencias del profesorado para mejorar la formación inicial, y es por ello que este tema no es nuevo en el ámbito de la investigación en Educación Física (Martínez, 1994; Sicilia, 1996).

\section{DISEÑO DE LA INVESTIGACION}

\subsection{OBJETIVOS DE LA INVESTIGACION}

a) Identificar las creencias del profesorado universitario de Educación Física en relación a los conceptos de "Cuerpo" y "Salud".

b) Describir las creencias del profesorado universitario de Educación Física en relación a los conceptos de "Cuerpo" y "Salud".

c) Analizar, interpretativamente, las creencias del profesorado universitario de Educación Física en relación a los conceptos de "Cuerpo" y "Salud".

\subsection{METODOLOGIA}

La presente investigación es un estudio cualitativo de carácter descriptivo y relacional. Se eligió trabajar la metodología cualitativa debido a que, en función de los objetivos de esta investigación, es necesario detectar cualidades, es decir, descripción, relaciones y desarrollo de características, prescindiendo de registros de cantidades, frecuencias de 
aparición o de cualquier otro dato reducible a números, realizándose la descripción de cualidades por medio de conceptos y de relaciones entre los mismos (Krause, 1995).

La metodología cualitativa se refiere a procedimientos que posibilitan una construcción de conocimientos sobre la base de conceptos. Desde aquí, la presente investigación en su carácter descriptivo y relacional utiliza una perspectiva inductiva, es decir, de lo particular a lo general, concretamente el procedimiento de codificación de la teoría fundada (Glaser y Strauss, 1967).

El trabajo de campo, una vez se transcribió, se sometió a análisis de contenido, apoyado en el programa computacional Atlas Ti 4.0. Los procedimientos de análisis empleados, en general se ciñeron a las propuestas de fragmentación y articulación de la "grounded theory" (Glaser y Strauss, 1967; Strauss y Corbin, 1994). Se realizó codificación abierta, con categorías emergentes; codificación axial, en torno a la agrupación de las categorías emergentes en familias de categorías; y codificación selectiva, mediante un trabajo interpretativo.

Este método permite obtener la información desde los mismos actores, sus significados y comprensiones del fenómeno, lo que se traduce en la utilización de las propias palabras de las personas, habladas o escritas (Taylor y Bogdan, 1984), lo que permitirá alcanzar una comprensión integrada de la materia de estudio e incorporar aquellos elementos subjetivos que son propios de la realidad del proceso estudiado.

\subsection{CARACTERISTICAS COMUNES DE LOS SUJETOS ENTREVISTADOS ${ }^{1}$}

Los sujetos entrevistados son profesores de educación física con experiencia profesional en los ámbitos de educación básica y media pero que en estos momentos se encuentran desarrollando su labor profesional como académicos de una carrera de Pedagogía en Educación Física, Deporte y Recreación, de una universidad privada de la ciudad de La Serena, en Chile, IV Región.

Chile es un país donde los profesores de educación física aún no han tenido una gran profesionalización, por lo que el desarrollo académico de los mismos se ha visto un poco limitado. Son pocos los profesores/as de educación física chilenos que poseen estudios de doctorado, por ejemplo, aunque ahora está emergiendo la necesidad de seguir perfeccionándose en el área.

De los 8 profesores entrevistados, 7 son hombres y 1 es mujer. Sólo dos (un hombre y una mujer) poseen título de Magíster, el resto son licenciados y ninguno es doctor, aunque uno de ellos está realizando los estudios conducentes al título de doctor.

Todos los profesores, a excepción de uno, superan la edad de 40 años.

\footnotetext{
A lo largo del trabajo se ha mantenido el anonimato de los sujetos que han participado de esta investigación. Y dado que este trabajo no se ha realizado a solicitud de la institución sino que ha sido una decisión personal, la institución a la que pertenecen los sujetos entrevistados tampoco será dada a conocer. Esto no quita que se haya intentado contextualizar y mostrar algunas características básicas de la misma.
} 


\subsection{CARACTERISTICAS DE LA ENTREVISTA EN PROFUNDIDAD}

La entrevista como técnica tiene como propósito que el investigador se sitúe en el lugar del entrevistado, suponiendo que la realidad de los otros es significativa, cognoscible y explícita (Goetz y Lecompte, 1988).

Teniendo en consideración lo anterior, por entrevistas cualitativas en profundidad se debe entender los reiterados encuentros cara a cara entre el investigador y los informantes, encuentros dirigidos hacia la comprensión de las perspectivas que tienen los informantes respecto de sus vidas, experiencias o situaciones, tal como las expresan sus propias palabras (Taylor y Bogdan, 1984).

\subsection{PROCEDIMIENTO}

El trabajo realizado se desarrolló en tres etapas:

a. Diseño y planificación del estudio. Incluye contacto con los profesores y elaboración de los guiones para las entrevistas en profundidad.

b. Implementación del estudio. Consistió en la realización de las entrevistas en profundidad.

Las entrevistas en profundidad se realizaron durante el mes de abril. Durante las entrevistas sólo nos encontrábamos el investigador y el entrevistado. Las entrevistas tuvieron diferentes duraciones pero rondaron, como tiempo promedio, los 60 minutos. Antes de iniciarse cada una, a cada entrevistado se le solicitó permiso para grabarla, accediendo todos a tal petición.

c. Análisis e informe de los resultados. Se realizó el registro de la información, su codificación y análisis descriptivo relacional de todas las entrevistas en profundidad. Para tal efecto se utilizó el siguiente proceso.

\subsection{PROCESO DE RECOLECCION Y ANALISIS DE LA INFORMACION}

Se realizaba una entrevista en cada día, lo que permitía que cada entrevista pudiera ser transcrita antes de comenzar la siguiente. Esto permitía ir levantando algunas categorías que pudieran ser contrastadas en próximas entrevistas.

Entre una entrevista y la siguiente, se iban realizando comparaciones constantes, hasta el momento en que los nuevos datos ya no agregaran información nueva.

Este proceso de análisis de datos se desarrolló siguiendo un procedimiento inductivo, utilizando como técnica el análisis de contenido, cuyo primer paso consiste en la codificación de los datos obtenidos, para luego dar paso a la construcción de árboles de categorías (Glaser y Strauss, 1967).

La codificación comprendió tres etapas: una codificación abierta que hizo emerger de los datos todas las categorías posibles. Una segunda codificación que implicó ir integrando los conceptos previos en unidades mayores (familias de códigos o categorías conceptuales). Por último, la codificación selectiva estableció relaciones entre las categorías conceptuales y la obtención de un modelo descriptivo y relacional del objeto de estudio, en este caso las creencias de los profesores/as universitarios acerca de los conceptos de cuerpo y salud. 
Durante el proceso de codificación y análisis, todos los datos fueron contrastados activamente con las preguntas directrices que se hicieron en esta investigación, por lo tanto, en el marco de todo lo indicado antes, se realizó la contextualización de los datos, lográndose categorías y subcategorías que se detallan en el análisis de los mismos.

\subsection{CRITERIOS ETICOS}

Los criterios éticos que hemos seguido en este estudio son los siguientes:

- Pactar con los participantes un acuerdo de participación donde se detallen los compromisos adquiridos por ambas partes

- Garantizar a los participantes el anonimato total en el informe

- Garantizar a los participantes la salvaguardia de sus intereses por encima de los que pudieran surgir de la propia investigación

\section{RESULTADOS}

Tras la codificación abierta y selectiva realizada con las entrevistas a los ocho docentes universitarios, las creencias del profesorado las podemos clasificar como sigue:

\section{a. Cuerpo máquina}

- Cuerpo con énfasis en la competición

- Cuerpo como instrumento de discriminación

- Cuerpo y valores: estereotipos sociales

b. Salud: limitada al sujeto

- Salud desde el modelo biomédico

- Salud desde el modelo psicoeducativo

- Promoción de la salud como ejercicio individual

\section{c. Educación física tecnocrática}

- Estereotipos de género

- Estrategias metodológicas

- Enseñanza de los valores: no planificación

- Profesional de la EF como enseñante, no educador

NOTA: a continuación podrán encontrar el modelo descriptivo y relacional surgido de esta investigación y con el que queremos mostrar las relaciones que hemos establecido entre los diferentes elementos emergentes que caracterizan a las creencias del profesorado universitario en relación a los conceptos de "cuerpo" y "salud". De más está decir que aunque la lectura de dicho modelo es de arriba hacia abajo, las relaciones son en ambas direcciones, por lo que unas características están influenciando en las siguientes y estas últimas condicionan a las anteriores. 


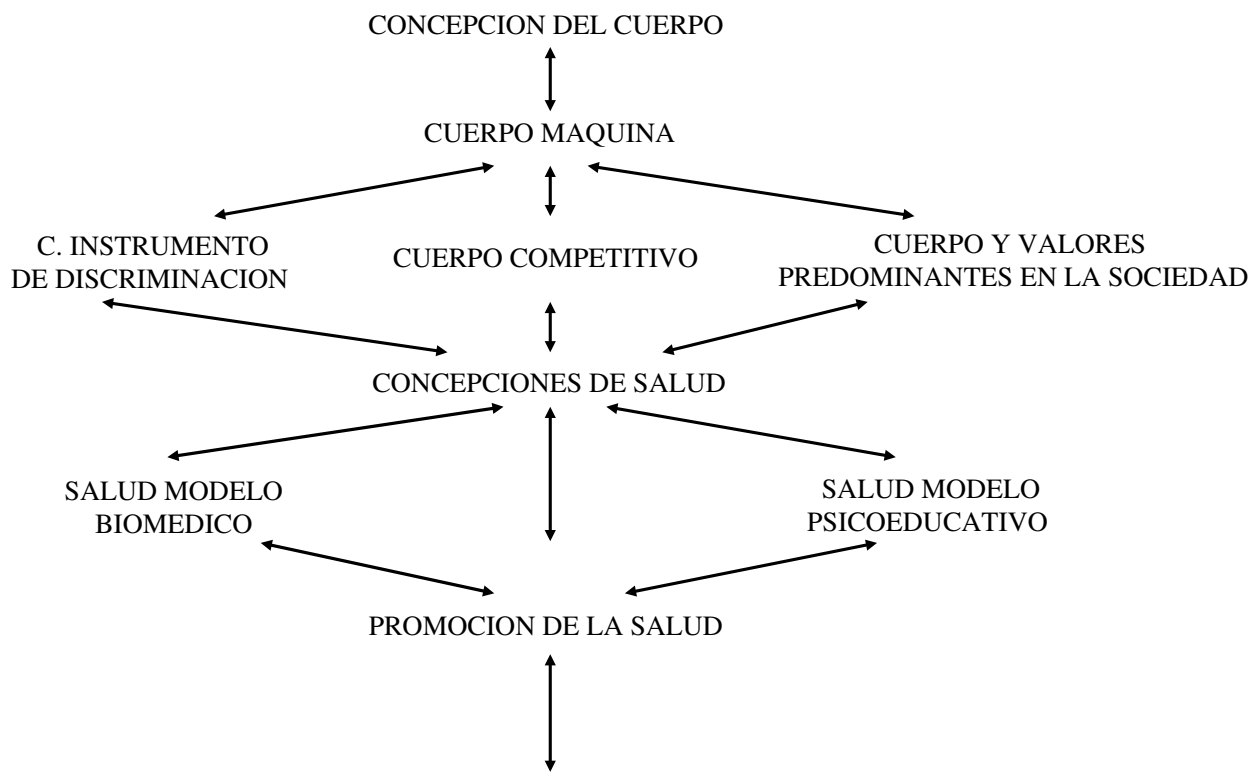

EDUCACION FISICA DESDE UNA PERSPECTIVA TECNICA

\section{a) Cuerpo máquina}

Como se puede leer en las citas textuales mostradas a continuación y obtenidas de las entrevistas realizadas a los docentes universitarios, ellos conciben el cuerpo como un entramado de piezas biofisiológicas ordenadas y que forman parte de un todo mayor que es la máquina, una máquina perfecta de movimiento. La noción biologicista del cuerpo se hace presente en las concepciones que se muestran, con las consecuentes propuestas metodológicas y formas de abordar los temas importantes de nuestra área, aspectos estos últimos que abordaremos a lo largo de este estudio.

"Para mí, el cuerpo es todo una máquina, una máquina perfecta con la cual nosotros realizamos todas las actividades de la vida diaria y todo lo que tiene que ver con el movimiento" (Entrev. 1; 63-66)

"Háblame del cuerpo, ¿en qué piensas cuando escuchas la palabra cuerpo". Una máquina de movimiento" (Entrev. 5; 50-51)

"El cuerpo es entonces una herramienta para desenvolverme en este mundo actual. Eso es lo que yo pienso que es el cuerpo. Es una herramienta para desenvolverme en este mundo actual. Mundo actual que tiene que ver con el mundo del trabajo, con el mundo de la familia, de la actividad física..." (Entrev. 7; 53-56)

Esta visión del cuerpo es propia de los docentes que se preocupan por el cuerpo y la salud en relación directa con la enfermedad, y no tanto por el sujeto que practica actividad física, pues consideran preponderante los aspectos orgánicos del cuerpo, del sistema al que ellos denominan máquina.

"Si lo vemos como un sistema, eso hace que si nuestro cuerpo anda bien, los eslabones de la cadena se van cumpliendo, creo que la salud tiene que ver con eso" (Entrev. 07: 63-65) 
- Cuerpo con énfasis en la competición

El cuerpo es un sistema que debe competir con otros, pues dicha actividad competitiva nos permite diferenciarnos de los otros/as que no practican actividad física de forma regular. Y para que el cuerpo rinda adecuadamente, debe ser cuidado para tal, atendiendo, nuevamente, al cuidado a los aspectos orgánicos del mismo.

"El cuerpo por sí compite" (Entrev. 6; 69)

"Hay ciertas tendencias en el desarrollo de las personas si es que yo no hago lo propio en función de lo que estoy transmitiendo, de tal forma que yo le digo a mis alumnos que descansen bien, duerman las horas que corresponden porque vamos a enfrentar un torneo la próxima semana" (Entrev. 2; 223-226)

\section{- Cuerpo como instrumento de discriminación}

Los profesores y profesoras universitarios estudiados parecieran no ser conscientes que con las concepciones que poseen en relación al cuerpo están valorando algunos aspectos de su profesión que pueden generar algún tipo de discriminación entre los discentes con los que comparten espacios y tiempos educativos.

\footnotetext{
"Entonces una de las problemáticas que yo tenía era esa, ¿cómo yo lograba que las niñas, en su grupo y su espacio lograban comportarse como mujeres y no como hombres? Es por eso que yo, cuando hacía las clases al ritmo de las mujeres se les ponía unas normas y a los varones otras. Las exigencias indudablemente eran iguales pero el gesto era diferente, se les enseñaba a ellas cómo tenían que caminar y por qué tenían que caminar así, porque ellas no pueden mover los brazos cuando caminan. Y se preguntaban por qué. Porque si usted no mueve los brazos la cadera se mueve y si usted mueve la cadera, cuando vaya a ser mamá, se case y tenga hijos, va a ver que eso le va a favorecer mucho, pero en la medida que usted cambie a un trote en el que mueva los brazos las caderas se van a empezar a achicar y va a tener problemas a la hora de tener hijos. Y además hay un concepto de trotar de una forma que es femenino y otro es masculino, y ustedes son femeninas, y eso hace que el acercamiento entre hombres y mujeres, las relaciones entre ellos sean mucho más exquisitos, más agradables, y a uno le agrada eso. Siento que hay una tendencia a eso, de que las niñas quieren ser como los hombres. No. Las niñas tienen que ser como ellas y tenemos que darles la posibilidad de que sean como ellas, no negarles sus posibilidades, y eso lo realizo en mis clases". (Entrev. 6: $180-195)$
}

¿Existen comportamientos de hombres y de mujeres? Sin duda que la falta de cuestionamiento de ciertas creencias sociales que estos profesionales muestran terminan generando situaciones discriminatorias entre sus alumnos y alumnas. El cuestionamiento de ciertas creencias y estereotipos no suele formar parte del trabajo educativo de los docentes entrevistados.

- Cuerpo y valores: estereotipos sociales

Es bastante habitual, en las entrevistas realizadas, encontrar comentarios en relación a la importancia de un modelo hegemónico de presentación personal, negando otras posibilidades en la forma de vestir y mostrar modelos de presentación de cuerpo diferentes 
a los habituales. Ante modelos diferentes no hay un trabajo educativo al respecto sino una negación de los mismos a través del tan mal usado reglamento de comportamiento que se realiza en los diferentes establecimientos educacionales, que en la mayoría de los casos son elaborados por las cúpulas directivas del mismo y no con la participación colegiada de todos los miembros de la comunidad educativa.

"Es relevante la presentación personal. Yo creo que pelo corto en ese sentido de los varones y las damas el pelo tomado... y ropa limpia que sea $a d$ hoc a la actividad que vamos a realizar..." (Entrev. 1: 125-127)

"Que es un poco el mensaje que se les da a los chicos acá, que se prohíban ciertas cosas a través del reglamento porque a quien queremos ver en el colegio eres tú, tú José Luis, tú eres el que está aquí, no está tu piercing, no está tu tatuaje, no está tu ropa...” (Entrev. 2: 316-319)

El cuerpo tiende a ser valorado como positivo cuando no se sale de la norma supuestamente correcta, aunque le estemos dando la espalda a una realidad social que descriptivamente es muy diferente a lo que un reglamento escolar pueda mostrarnos como realidad. Una realidad social en donde las diferencias en cuanto a forma de vestir, opinar, de gustos, preferencias, etc., es el común denominador.

\section{b) Salud limitada al sujeto}

- $\quad$ Salud desde el modelo biomédico

"Un ejemplo. Una buena condición física es aquella persona que es capaz de correr $12 \mathrm{~km}$, una buena capacidad física en cuanto a la variable resistencia ¿Cómo lo corres? Sin parar, solamente al trote. Uno se puede demorar una hora, otro 2 horas, pero debes hacerlo sin parar y si lo logras sin parar, aunque vayas muy lento, para mí eso habla de una buena condición física. El que no llega es porque no tiene la condición física aún para esa variable. El tiempo es de uno, y cuando tú logras eso sin duda que ha habido cambios internos en tu parte fisiológica, hay cambios que te permiten por ejemplo mejorar tu capacidad cardiaca, el corazón te crece más, tener una mayor calidad de tus movimientos, te sientes más sólido, no te sientes cansado... y en definitiva eso es estar saludable" (Entrev. 6: 54-62)

Entender la salud desde el punto de vista biomédico es algo bastante característico de los docentes universitarios con los que se ha realizado este estudio. Dado esto, es común centrar el trabajo pedagógico en el área de la educación física a partir del desarrollo de la condición física, entendiendo, equivocadamente por cierto, que aumentar la condición física está directamente relacionado con el mejoramiento de la salud ${ }^{2}$. Se entiende, entonces, que la salud es sinónimo de un nivel de condición física determinado, por lo que se limita el concepto de salud a la vertiente biológica, y se niega la vertiente psicológica y social del concepto, como ya hemos comentado en el marco referencial de este trabajo.

2 Para profundizar en esta temática, ver estudios e investigaciones de los académicos José Devis Devis y Carmen Peiró Velert, de la Universidad de Valencia, España. 
- Salud desde el modelo psicoeducativo

Algunos docentes entrevistados, aunque sólo dos de los ocho sujetos estudiados, amplían su mirada del concepto de salud a la vertiente psicológica del sujeto, por lo que la perspectiva ya no es única y exclusivamente biológica, sino que entienden que los aspectos emocionales son relevantes en un estado de bienestar determinado.

"Y lo otro es la línea más mental y más espiritual que es eso que no se ve, pero también debemos cuidar nuestras emociones, nuestro estrés, buscar espacios que te permitan tener recreación, tener distracción, priorizar en la vida, contacto con la naturaleza, contacto con compartir con familia, con hijos" (Entrev. 8: 104-107)

\section{- Promoción de la salud como ejercicio individual}

Dada la limitación del concepto de salud al ámbito biológico y psicológico, y olvidando la influencia del contexto social en el que los sujetos viven diariamente y de cómo éste condiciona el estado de bienestar de una persona, es lógico percibir cómo el concepto de salud es tratado desde el punto de vista individual. En la cita siguiente podemos ver cómo la obesidad, por ejemplo, es una enfermedad que para ser solucionada debe venir acompañada, casi exclusivamente, de una promoción de la salud basada en el aumento de información que los diferentes sujetos deben tener, entendiendo que a mayor información acerca de la temática menores problemas de obesidad.

"Atacando de lleno la obesidad en los colegios... impartiendo clases teóricas sobre nutrición y las consecuencias de la misma y los beneficios, o sea, informando más a los niños y niñas de nuestro país" (Entrev. 4: 58-60)

\section{d. Educación física tecnocrática}

Dado todos los comentarios realizados más arriba, y como consecuencia de ello, la educación física es entendida por los docentes universitarios desde una perspectiva técnica de la educación, y por tanto nuestra área se convierte en una asignatura del currículo escolar cuya última finalidad es trabajar casi exclusivamente la parte física de los discentes, y por tanto la labor del profesional de la educación física es atender los aspectos biológicos del cuerpo y el movimiento.

"Educación Física es educar la parte física... yo como profesor enseñarle a los alumnos a que ellos sepan desarrollarse físicamente y todo el ámbito de lo que es nuestro cuerpo y en relación a todo lo que... al movimiento". (Entrev. 1: 10-13)

Es el modelo de estimulos-respuestas y la repetición mecánica de las tareas motrices el eje central de la labor pedagógica de los profesionales de nuestra área. El aumento del rendimiento físico es sinónimo de aprendizaje motor, con las consecuentes limitaciones formativas de la educación física escolar.

"Ese se le deja al final, se le da otras oportunidades. Se le dice mire, esta nota, tiene 6 estímulos para que los trabajes y mejore tu capacidad de subir la cuerda, pues bien, a ti te costó, pero yo te espero hasta el final de semestre. Si tú vienes al gimnasio, te preparas y 
me preguntas cómo mejorar eso, yo te voy a decir, mira, esta es la forma de estimular tus músculos y hacerlo. Tal día vas a tener la cuerda, tal día vas a tener todas las condiciones pero hazlo. Y me ha tocado alumnos que al final de año me dicen profe, yo quiero subir la nota porque por ejemplo en velocidad no logré el tiempo adecuado, pero me entrené, mejoré, quiero mejorar mi nota, y si consiguió mejor tiempo pues yo le borro la nota del principio, y además le pongo la actitudinal, que es la solidaria. Para mí la nota no es algo que se quedó en el libro y no se borró más. Y no tengo "atados" con las evaluaciones, si el alumno demuestra que mejoró, pues ningún problema”. (Entrev. 6: 229-239)

- Estereotipos de género

"Sí, yo respeto mucho a las mujeres, no sé si seré machista, creo que no aunque todos somos un poquito machista y feminista al mismo tiempo, pero creo que desde mi perspectiva y mi historia de 15 años de profesor, me ha mostrado que a las mujeres les falta como esa energía, como pocas mujeres que existen". (Entrev. 7: 98-101)

La concepción mecanicista, repetitiva y tradicionalista del área de educación física es impregnada también por una serie de estereotipos propios de dicha visión y perspectiva tradicionalista del área, como son los estereotipos de género. El profesional hombre es mejor considerado que la profesional mujer, pues desde la concepción del cuerpo máquina y competitivo, la condición física de la fuerza y el modelo deportivo hacen pensar que el hombre posee mejores y mayores capacidades que las mujeres.

\begin{abstract}
"Ahora en el deber ser, eeee en el deber ser, bueno creo que hay ciertas condiciones de género dada las características de la clase en que ya los comentamos en preguntas anteriores, que hacen que el hombre tenga una mayor probabilidad de éxito que una profesora dama, digamos ahora en la orientación en general en Chile se está viendo que progresivamente en la medida de las posibilidades las clases se han ido separando, excepto los cursos menores, cierto, se han ido separando, profesora con damas y profesor para varones, en general en la orientación de la educación privada y particular subvencionada también, y eso tiene que ver con este manejo mmmm, con este manejo yo creo que se le hace más difícil a una profesora el manejo de los cursos numerosos en una escuela con espacios muy abiertos de cursos mixtos o de grupos de varones solamente. Entrev. 7: 105-116)
\end{abstract}

\title{
- Estrategias metodológicas
}

La educación física entendida desde una perspectiva pedagógica técnocrátrica genera una serie de estrategias metodológicas basadas en la ejecución de ciertas técnicas deportivas, y nos conduce a entender que dicho aprendizaje basado en el esfuerzo y la repetición puede ser transferido a la vida real de los alumnos/as, concibiendo que existe una traspolación directa entre lo acometido en clase de educación física y la cotidianeidad de los sujetos.

"Entonces tú tienes que llegar allá y nadar y nadar y vas a tener que nadar, o sea, yo creo que lo hago de una manera inconsciente que ellos tienen que siempre ir superándose, superándose y al final terminan nadando y la mayoría terminan haciendo mil metros que ése es el examen final ¿Cómo lo hacen?, no sé, una buena técnica, una técnica más o menos pero la tienen que hacer y eso les ayuda en el fondo a eee eso es una norma de vida, no trabarte con un problema que tengas enfrente sino tení que seguir, la vida te va a poner muchas dificultades. 
Sin agobio no hay vida, hay que hacérselo saber, tiene que seguir si la vida funciona igual no más" (Entrev. 4: 417-425)

\section{- Enseñanza de los valores: no planificación}

Efectivamente, hay docentes entrevistados que en su discurso enfatizan la importancia del trabajo valórico en el área de la educación física, aunque todos coinciden en señalar que dicho trabajo no está planificado y sistematizado, sino que emerge de la práctica diaria en el patio escolar. Además de la no planificación del trabajo de los valores, el trabajo de los mismos se limita, casi exclusivamente, a conversar en relación a los valores, lo que no permite generar espacios y tiempos en donde esos valores puedan ser practicados, experimentados, cuestionados, debatidos, etc.

"Por ejemplo, los valores generalmente, no tengo un sistema muy ordenado para trabajarlos sino que siempre estoy conversándolo con ellos, hablándolos con ellos, de por qué es importante, de por qué se tienen que respetar. Mucho a través de conversaciones directas con ellos, con el grupo y de forma individual, porque no tengo sistema muy específico así para eso". (Entrev. 3: 77-81)

"En mí generalmente es así, aunque a pesar de que uno tiene claro que tiene que ir viendo, pues hay momentos que son claves y que se producen para hablar de un determinado valor, que se producen en la clase, y si no se producen uno tiene que ir viendo, de acuerdo a los objetivos que se va planteando, cómo puede ir desarrollando ese valor". (Entrev. 3: 85-88)

- $\quad$ Profesional de la EF como enseñante, no como educador

Desde la limitación del entendimiento de la disciplina escolar de Educación Física desde una perspectiva tecnocrática, es entendible que la capacidad del profesor de educación física para ejecutar correctamente las actividades que propone sea de gran relevancia, pues así el docente podrá y deberá convertirse en un modelo de comportamiento motor que debe ser seguido por los alumnos y alumnas. Además de ello, se supone que el profesional del área es una persona que 'entrega' conocimientos, lo que sin duda imposibilita la construcción autónoma y crítica del mismo por parte del alumnado.

"Un profesional de la educación física es una persona que sepa realmente lo que está entregando" (Entrev. 1: 17-18)

"Que el alumno logre aprender todo lo que el profesor le entrega" (Entrev. 1: 20)

"Sería algo como normal como predicar con el ejemplo, yo no puedo decirle a un chico oye no te puedes colocar un piercing si tú tienes un piercing o córtate el pelo si tú tienes el pelo largo..para mí es predicar con el ejemplo ahh". (Entrev. 1: 133-136)

\section{INTERPRETACION DE LOS RESULTADOS}

"El cuerpo es un tema que se presta especialmente para el análisis antropológico ya que pertenece, por derecho propio, a la cepa de identidad del hombre. Sin el cuerpo, que le proporciona un rostro, el hombre no existiría. Vivir consiste en reducir continuamente el mundo al cuerpo, a través de lo simbólico que éste encarna" (Le Breton, 1995: 7) 
El estudio acerca de las creencias, ya sean éstas explícitas e implícitas, que posee el profesorado universitario de educación física lo consideramos de principal importancia porque, de alguna manera, dichas creencias guían y orientan su conducta, ya que la mera observación de práctica no puede explicar, por sí sola, los procedimientos docentes, y consideramos que este marco de creencias es desde el que se comprenden e interpretan las experiencias que se viven y desde las que se actúa racionalmente en el ámbito pedagógico (Clark y Peterson, 1990).

Un primer aspecto que nos parece de relevancia es que al contrario de lo que se pueda suponer, y de lo que señalan algunos estudios (Gómez, 2003), las creencias del profesorado estudiado se alejan del discurso actual de la educación física, más basada en perspectivas constructivistas y dialógicas de los procesos educativos que en perspectivas tecnocráticas.

Esta perspectiva tecnócratica está anclada en una primera creencia eje que es la concepción del cuerpo como un "cuerpo máquina". Desde esta metáfora, el cuerpo es entendido como una realidad biológica y es por ello que las creencias de los docentes en relación al concepto de salud tienen relación con una acepción del término salud como el funcionamiento adecuado de los diferentes sistemas orgánicos.

"Esta perspectiva objetivo-biologicista puede servir para explicar muy gráficamente algunos aspectos relacionados con nuestra parte material, aunque olvida otros que son fundamentales para comprender la complejidad del cuerpo en su globalidad. Por ejemplo, aunque la idea del cuerpo-máquina deja clara muchas cararacterísticas del cuerpo en el que vivimos, no aclara porque no siempre vivimos nuestro cuerpo de la misma manera. Muchas sensaciones, sentimientos, emociones y recuerdos dejan una huella somática, a veces más explícita que aquello que podemos comunicar con palabras. Cuando afirmamos que nos sentimos mal o que no nos gustamos, hacemos referencia a un conjunto de sensaciones, entre las que destaca qué sentimos hacia nuestro cuerpo, o dicho de otro modo, cómo nos sentimos en él” (Devís, 2000: 63)

Desde esta visión, todos los cuerpos deben ser iguales y es por ello que nuestros profesores/as, en algunas ocasiones caen en situaciones de discriminación en su labor pedagógica, pues los cuerpos diferentes son invisibilizados cuando no obligados a ser de forma diferentes a como naturalmente se presentan. La tendencia de la lógica dicotómica escolar a la homogeneización se hace presente, también, en los docentes universitarios con los que hemos trabajado. La aceptación de las diversidades corporales está aún lejos de ser una idea central en el trabajo de los docentes con los que hemos trabajado. Aceptar la diversidad y trabajar a partir de ella significa romper con la creencia de que en el ámbito educativo todos debemos ser y parecer iguales.

“... el reconocimiento de la diversidad del ser humano exige la primera ruptura que ha de realizar la escuela contra la homegeniedad en sus aulas. Esa ruptura supone por un lado, una tolerancia activa, es decir, un esfuerzo y un interés por comprender al otro como es y, por otro, la ruptura con el deseo permanente en nuestras aulas de clasificación y sometimiento de las personas a una norma. Es una ruptura contra el orden que supone la homegeneidad y, a la vez, el reconocimiento de la cualidad más natural del ser humano: la diferencia. Lo normal es la diferencia y no la homogeneidad. De la misma manera que no existen dos amapolas iguales, no existen dos personas iguales" (López, 2003: 31) 
En esta situación, nuestro cuerpo se halla constreñido por una lógica escolar que lo limita a los espacios y tiempos propios de la institución educativa formal, marcada por reglamentos y normas no colegiadas y que deben ser cumplidas por todos. Pareciera entonces que el cuerpo se convierte en un instrumento de adoctrinamiento conductual.

\begin{abstract}
"Nuestro cuerpo, situado en el espacio y en el tiempo escolares, se halla constreñido al cumplimiento de normas que no consideran sus características y sus exigencias. Esto tiene diversas consecuencias, a saber: se consigue que los escolares aprendan que no pueden aprender, que deben controlar su cuerpo y sus inquietudes, que son malos para tal o cual asignatura, etc. Revertir estas circunstancias es lento, y, las más de las veces, improductivo" (Calvo, 2005: 94).
\end{abstract}

Se convierte el cuerpo, entonces, en una realidad únicamente física, lo que conlleva a considerarlo como una realidad que se puede medir, manipular, controlar, independientemente del sujeto persona. Según Barbero (2007: 22), desde esta perspectiva "el cuerpo será tan dócil como cualquier otro objeto inerte".

Es importante mencionar que esta concepción del cuerpo como una máquina no es algo propio del siglo XXI, sino que emerge con fuerza en Galileo y su revolución galileana, en donde las nociones de medida, exactitud, rigor, etc., cobran excesiva importancia.

Como consecuencia de ello, el sujeto se encuentra dividido en dos realidades diferentes, el cuerpo y el alma. Es por ello que Descartes llegara a afirmar que el pensamiento era totalmente independiente del cuerpo.

Esta concepción del cuerpo como cuerpo máquina (Le Breton, 1995; Devís, 2000; Samaniego y Sánchez, 2001; Barbero, 2007; Sparkes, 2001; Kirk, 2007) conlleva a entender otra área primordial de la educación física y el deporte, como es la salud, de forma coherente con ese cuerpo máquina. Eso significa estar anclado, para trabajar la salud, en un modelo puramente biomédico, y eso representa que atenderá casi exclusivamente al desarrollo de la condición física, aceptando que un aumento de ésta conduce, irremediablemente, a una mejor salud. Esto, a pesar de las numerosas voces que se alzan en contra de dicha perspectiva y que nos muestran que los beneficios para la salud no están tanto en el mejoramiento de los niveles de condición física sino en una práctica de actividad física autónoma, disfrutada socialmente, autogestionada...

"Enfatizar la actividad física en lugar de la condición física es mucho más considerado con todas las personas, especialmente con las más necesitadas de los beneficios saludables de la práctica. Fundamentarse en el desarrollo de la condición física para promocionar la salud ha tenido muchos problemas porque, por ejemplo, dificultaba que las personas con poca disposición para realizar ejercicios se implicaran en prácticas físicas y que las personas con una pobre condición física u obesas abandonaran este tipo de programas antes de alcanzar los umbrales mínimos que se consideraban adecuados" (Devís y Beltrán, 2007: 33)

En nuestro estudio es el modelo biomédico de salud el que tiene mayor presencia entre las creencias de los docentes estudiados, lo que significa que ellos ponen el énfasis en una práctica de la educación física para mejorar la salud entendiendo que ésta es sinónimo de no tener enfermedades y lesiones. Es por ello que para los profesores universitarios se hace importante el visitar periódicamente al médico para prevenir problemas de salud. 
Esta es la visión preponderante durante todo el siglo XIX y la mayor parte del siglo $\mathrm{XX}$, en donde "la concepción sobre el proceso de salud-enfermedad estuvo dominada por la visión positivista del enfoque biomédico, o bien por la perspectiva socio-sanitaria de la medicina social, las cuales concebían la salud como ausencia de enfermedad" (Castro, 2007: 13)

Esta visión mecanicista de la salud lleva a que los aspectos realmente importantes a trabajar en el área de educación física sean muy limitados en la formación de los sujetos escolarizados, y atiendan principalmente a:

\begin{tabular}{|l|l|}
\hline Resistencia cardiorrespiratoria & Resistencia y Fuerza muscular \\
\hline Flexibilidad & Composición corporal \\
\hline Velocidad & Potencia \\
\hline Agilidad & Equilibrio \\
\hline
\end{tabular}

Todos estos aspectos son importantes pero muy limitantes en la formación de un sujeto que pretendemos emancipado y autónomo para desenvolverse en la vida social democrática y en su cotidianeidad particular.

\section{REFLEXIONES FINALES A MODO DE CONCLUSION}

Más que conclusiones definitivas tras este acercamiento investigativo a las creencias del profesorado universitario de educación física en relación a los conceptos de "Cuerpo" y "Salud", nos gustaría mostrar algunas reflexiones que emergen del mismo y que pensamos, pueden ayudar a seguir avanzando en relación a la problemática estudiada.

a) Pareciera sorprendente que, a pesar del gran desarrollo de la educación física como disciplina académica ${ }^{3}$, profesores universitarios aún mantenga aquellos estereotipos totalmente acordes con una visión de la Educación Física muy tradicionalista basada, exclusivamente, en el deporte de carácter competitivo y la condición física.

b) Es importante reflexionar que lo que partió como un estudio en donde se investigarían las concepciones implícitas del profesorado, bajo el entendido que los docentes universitarios a estudiar mantendrían un discurso alineado con la reforma educacional chilena, más acorde con planteamientos constructivistas (no todavía dialógicos) de la educación, terminó siendo un estudio sobre creencias, pues en el propio discurso y de forma explícita fueron apareciendo lo que un principio suponíamos que sólo aparecería de forma subrepticia en las palabras enunciadas.

c) Este primer acercamiento a las creencias del profesorado universitario sobre los conceptos de "Cuerpo" y "Salud" sería recomendable poder repetirlo en otras comunidades de académicos universitarios chilenos, con la intención de ir generando una

3 Como botón de muestra resulta sugerente explicitar algunas de las publicaciones periódicas de carácter científico técnico más importante del área de la educación física: Quest; European Physical Education Review; Journal of Teaching in Physical Education; Sport, Education and Society; Physical Education and Sport Pedagogy 
producción científica que permitiera trabajar sobre dichas creencias y modificarlas, pues esa es una de las formas de ir modificando una práctica escolar alejada totalmente de una educación física tradicional y que entienda que la autonomía de los sujetos, la convivencia democrática, la aceptación y disfrute de la diversidad y el valor del diálogo no son elementos propios de otras asignaturas del currículo escolar, sino que es la única forma de mejorar la salud, en su visión más amplia, de los niños y niñas con los que trabajamos.

\section{BIBLIOGRAFIA}

Barbero, J. (2007). Capital (es) corporal (es) que configuran las corrientes y/o contenidos de la educación física escolar. En Ágora para la Educación Física y el Deporte, 4-5; 21-38.

Calvo, C. (2005). Entre la educación corporal caótica y la corporalización escolar ordenada. Revista Iberoamericana de Educación, 39, 91-106.

Castro, J. (2007). "Los paradigmas sobre el proceso salud-enfermedad y sus consecuencias en la educación para la salud". En Diana del Pilar Cevallo Lugo y Nery Cecilia Molina Restrepo (Editoras) Educación corporal y salud: gestión, infancia y adolescencia. Funámbulos Editores. Universidad de Antioquia, Colombia. Pp. 13-28.

Clark, C. y P. Peterson (1986) "Procesos de pensamiento en los docentes". En Merlin Wittrock. La investigación en la enseñanza III. Profesores y alumnos. Paidós. Barcelona. Pp. 443-539.

Contreras, O. y otros (2002). Las creencias en la formación inicial del profesorado de educación física. Incidencias en la transformación de su pensamiento. Revista Interuniverstiaria de Formación del Profesorado, 45, 131-149.

Devís, J. y V. Beltrán (2007). "La educación física escolar y la promoción de la actividad física y la salud". En Diana del Pilar Cevallo Lugo y Nery Cecilia Molina Restrepo (Editoras) Educación corporal y salud: gestión, infancia y adolescencia. Funámbulos Editores. Universidad de Antioquia, Colombia. Pp. 29-43.

Devís, J. y C. Peiró (2001). "Fundamentos para la promoción de la actividad física relacionada con la salud". En José Devís Devís (coord.) La Educación Física, el Deporte y la Salud en el siglo XXI. Editorial Marfil. Alicante. Pp. 295-321.

Devís, J. (2000). Actividad física, deporte y salud. Inde. Barcelona.

Glaser, B. y A. Strauss (1967). The discovery of grounded theory. Strategies for qualitative research, Chicago: Aldine Publishing Company.

Goetz, J. y M. Lecompte (1988). Etnografía y diseño cualitativo en investigación educativa. Editorial Morata. Madrid.

Gómez, L. (2003). Las teorías implícitas de los profesores y sus acciones en el aula. Ponencia presentada al VII Congreso Nacional de Investigación Educativa, Guadalajara, México.

Kirk, D. (2007). Con la escuela en el cuerpo, cuerpos escolarizados: la construcción de identidades inter / nacionales en la sociedad postdisciplinaria. En Ágora para la Educación Física y el Deporte, 4-5; 39-56.

Krause, M. (1995). La investigación cualitativa: un campo de desafíos. En revista Temas de Educación, 7, 66-87.

Le Breton, D. (1995). Antropología del cuerpo y modernidad. Nueva Visión. Buenos Aires.

López, M. y otros. (2003). Conversando con Maturana de educación. Ediciones Aljibe, Málaga.

Martínez, L. (1994). Influencia de las creencias implícitas en la formación inicial de los especialistas en EF. En S. Romero Granados (coord.) Actas del I Congreso Nacional de EF de Facultades de Ciencias de la Educación y XII de Escuelas Universitarias de Magisterio. Sevilla, Universidad. 
Pérez, V. y R. Sánchez (2001). Las concepciones del cuerpo y su influencia en el currículum de la Educación Física. Revista Digital efdeportes.com, 6, 33. Web visitada (http://www.efdeportes. com/efd33a/cuerpo.htm) el 8 de mayo de 2008, 9.03 horas.

Sicilia, A. (1996). El profesor de EF en Andalucía. Cómo piensa, califica y desarrolla sus contenidos y actividades. Habilidad Motriz. Revista de Ciencias de la Actividad Física y el Deporte, 8, 34-55.

Sparkes, A. (2001). "Las identidades deportivas y el cuerpo: una relación problemática". En José Devís Devís (coord.) La Educación Física, el Deporte y la Salud en el siglo XXI. Editorial Marfil. Alicante. Pp. 181-201.

Strauss, A. \& J. Corbin (1994). Grounded Theory methodology: An overview, In: Handbook of Qualitative Research (Denzin, N., K. and Lincoln, Y.,S., Eds.). Teoría metodología: Una visión general, En: Manual de la investigación cualitativa (Denzin, N., K. y Lincoln, Y., S., Eds.). Sage Publications, London, 1-18. Sage Publications, Londres, 1-18.

Taylor, S. y R. Bogdan (1984). Introducción a los métodos cualitativos de investigación. Paidós. Barcelona. 
